\title{
Adaptive Sliding Mode Dynamic Positioning Control for a Semi-Submersible Offshore Platform
}

\author{
Dongya Zhao ${ }^{1}$, Shouli Gao ${ }^{1}$, Sarah K. Spurgeon ${ }^{2}$ and Markus Reichhartinger ${ }^{3}$
}

\begin{abstract}
In this paper, an adaptive sliding mode dynamic positioning control approach is proposed for a semi-submersible offshore platform. The actuator dynamics are slow and thus a first order sliding mode control approach is used to maximise tracking accuracy in the presence of typically unmodelled actuator dynamics. The sliding mode control is designed with an adaptive feedback gain to counter the effects of model uncertainty and external disturbances such as the waves. The control implementation uses a sliding mode differentiator for online estimation of velocity and acceleration. The stability of the system is analyzed using Lyapunov methods. The control algorithm is validated using illustrative examples.
\end{abstract}

\section{INTRODUCTION}

The semi-submersible offshore platform (SSOP) is an important element in the offshore oil and gas industry. A key issue in SSOP operation is dynamic positioning (DP), whereby the SSOP maintains its desired position and heading by control of the propulsion system in order to suppress environmental disturbances induced by the waves, currents, and wind [1]. When compared with other positioning methods, DP has advantages for the SSOP where the platform will typically operate in deep sea with requirements for high positioning accuracy and high maneuverability. This control problem is thus receiving current attention in the literature [2].

DP control development has seen three stages [3]. Initially, control of a three degree of freedom SSOP was carried out using PID control [4]. It proved difficult to select appropriate PID control parameters due to the complex dynamics of the SSOP. Then, optimal control was combined with Kalman filtering to improve performance [5]. However, the optimal control and Kalman filter require accurate dynamic models in the design procedure, which may be difficult to obtain in practice. Finally, some advanced DP control algorithms have been developed for the SSOP including predictive control [6], robust adaptive control [7], passivity based control [8], back stepping control [9] and sliding mode control (SMC) [10].

\footnotetext{
*This work is partially supported by the National Nature Science Foundation of China under grant nos 61473312 and the Chang Jiang Scholar Program.

${ }^{1}$ Dongya Zhao and Shouli Gao are with the College of Chemical Engineering, China University of Petroleum (East China), Qingdao 266580, China dongyazhao@139. com

${ }^{2}$ Sarah K Spurgeon is with the Department of Electronic and Electrical Engineering, University College London, Torrington Place, London WC1E 7JE, United Kingdom s. spurgeonducl . ac . uk

${ }^{3}$ Markus Reichhartinger is with the Institute of Automation and Control, Graz University of Technology, Inffeldgasse 21B/I 8010, Graz, Austria markus.reichhartingeretugraz.at
}

Among these advanced control approaches, SMC is an attractive solution due to its simple control structure, systematic design procedure, rapid response and inherent insensitivity to external disturbances. The application of SMC for DP of a turret moored floating production storage and offloading (FPSO) system was studied in [11]. A SMC was designed and experimentally tested for tracking control of underactuated autonomous surface vessels [12]. The effectiveness and superiority of SMC for DP system was validated experimentally in [10]. Super twisting control, a second order SMC algorithm, was also applied to surface vessels and was shown to deal with parameter variations effectively [13].

It has been seen that SMC can achieve good performance for DP systems. There are some areas which need further attention to fully assess the effectiveness of the paradigm. One area is the impact of actuator dynamics on the process. The second is the area of reconstructing unmeasurable states. The final area is that of controller tuning for what is a highly uncertain system. It has been shown [14] that the higher the order of the homogeneous SMC, the less sensitive it is to the presence of unmodelled but 'fast' actuators. More recent contributions [15], [16] have seen the debate around performance of SMC of various types in the presence of actuator dynamics intensifying. The general consensus is that for 'fast' actuator dynamics, a higher order SMC may be expected to give better performance in the presence of unmodelled actuator dynamics than a first order SMC, but that when unmodelled actuator dynamics are 'slow' a first order SMC may be better [16]. In this paper we assume a simple first order model for the actuators and adopt a first order SMC approach as the actuator dynamics for a SSOP are relatively slow and the assumed actuator model is a simplification of the actual actuator dynamics. The adopted first order SMC approach requires measurement of the velocity and acceleration which is difficult in practice. To overcome this problem, a state observer, such as high gain state observer [17], passivity state observer [18] and sliding mode observer [19] have been designed to estimate velocity. However system observers require the use of dynamic models during the design process. In this paper, a novel robust differentiator [21] is used for controller implementation. This differentiator has been implemented in a toolbox and can estimate velocity and acceleration simultaneously online without requiring a dynamic model [20]. In SMC design, the bounds of the uncertainty and external disturbance are frequently required during the controller design process. In this paper an adaptive law is used which can update the feedback control gain online without a priori knowledge of 
the bounds [22], [23].

The main differences between the proposed approach and the existing literature on SMC for DP of SSOP [10], [11], [12], [13] can be summarized as: (1) the proposed approach considers the impact of both modelled and unmodelled actuator dyanmics on the SMC performance; (2) a robust differentiator is employed to estimate the velocity and acceleration online, which renders the control algorithm more readily applicable; (3) an adaptive control feedback gain is designed to deal with the effects of controller tuning in the presence of external disturbances. The paper is organized as follows: in Section 2, the problem is formulated. In Section 3 , the proposed control algorithm is developed and the corresponding stability analysis is given. In Section 4 , case studies are demonstrated to validate the proposed approach. Finally, in Section 5, some conclusions are drawn.

\section{Problem Formulation}

The kinematic and dynamic model of the SSOP is given by [24]

$$
\begin{gathered}
\dot{\eta}=J(\psi) v \\
M \dot{v}+D v=\tau+d \\
J(\psi)=\left[\begin{array}{ccc}
\cos (\psi) & -\sin (\psi) & 0 \\
\sin (\psi) & \cos (\psi) & 0 \\
0 & 0 & 1
\end{array}\right]
\end{gathered}
$$

where $J(\psi)$ is the Jacobian matrix, $J^{-1}(\psi)=J^{T}(\psi)$ for all $\psi, \eta=[x, y, \psi]^{T}$ is the position vector in the Geodetic coordinate system in which $x y \psi$ denote $X, Y$ directions and the angle of rotation of the bow around $Z$ respectively, $\nu=$ $[u, v, r]^{T}$ is the velocity vector in the ship coordinate system in which $u, v, r$ denote to the surge, sway and yaw modes of a SSOP, $M, D \in R^{3 \times 3}$ represent inertia and linear damping matrices respectively, $\tau \in R^{3 \times 3}$ represents the control forces in surge and sway, and moment in yaw, $d \in R^{3 \times 3}$ represents the disturbance caused by waves, currents, and winds.

In light of (3), the dynamic equation can be written as

$$
P(\eta) \ddot{\eta}+Q(\eta, \dot{\eta}) \dot{\eta}=\tau(t)+d(t)
$$

where $P=M J^{-1}(\psi)$ and $Q=M \dot{J}^{-1}(\psi)+D J^{-1}(\psi)$. Note that $\|P\|$ is bounded and invertible, because $M$ is a positive definite constant matrix and $J^{-1}(\psi)$ is an invertible matrix.

Assumption 1: $\|d(t)\| \leq d_{0}$ and $\|\dot{d}(t)\| \leq d_{1}, d_{0}>0$ and $d_{1}>0$ are positive constants.

Assumption 2: $\eta$ can be measured.

Remark 1: $d$ is the low frequency disturbance caused by the waves, currents, and winds. The high frequency portion will not be considered in the control design because in general the SSOP system has the ability to suppress high frequency signals.

A robust adaptive SMC will be developed for the SSOP whose structure is given in Fig 1. A differentiator is used to estimate $\dot{\eta}$ and $\ddot{\eta}$, an adaptive law is designed to update feedback control gain which will be detailed in the following

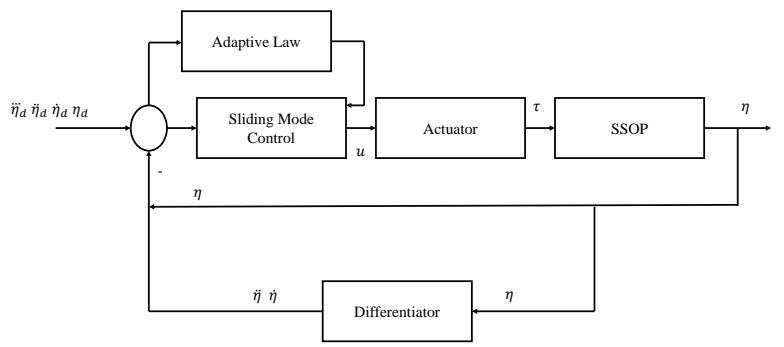

Fig. 1. The system block diagram of the adaptive sliding mode dynamic position control scheme

context, $u \in R^{3}$ denotes the input to the actuators, $\tau \in R^{3}$ is the control signal experienced by the platform.

Remark 2: For the controller design the actuators are approximately modelled by a first order lag. The actuator dynamics for the SSOP are slow and thus to enhance the performance in the sliding mode, a first order sliding controller is considered [15], [16].

The transfer function of the assumed actuator model is given by

$$
G_{a c t}(s)=\frac{\lambda}{s+\lambda}
$$

where $\lambda=1 / T, T>0$ is the time constant. According to (5), the relationship between $u$ and $\tau$ is given by

$$
\dot{\tau}+\Lambda \tau=\Lambda u
$$

where $\Lambda=\operatorname{diag}\left\{\left[\begin{array}{lll}\lambda_{1} & \lambda_{2} & \lambda_{2}\end{array}\right]\right\}, \lambda_{i}>0, i=1,2,3$. In light of (4) and (6), the system dynamic equation assumed for the controller design can be rewritten as

$$
P \dddot{\eta}+\dot{P} \ddot{\eta}+Q \ddot{\eta}+\dot{Q} \dot{\eta}+\Lambda P \ddot{\eta}+\Lambda Q \dot{\eta}=\Lambda u+\Lambda d+\dot{d}(7)
$$

Assumption 3: $\left\|P^{-1}\right\|\|\dot{d}\|+\left\|P^{-1} \Lambda\right\|\|d\| \leqslant \gamma_{0}, \gamma_{0}>0$ is a positive constant.

Remark 3: According to Assumption 1, the properties of $P$ and the definition of $\Lambda$, Assumption 3 is reasonable.

A robust adaptive SMC for DP of the SSOP (7) will be designed incorporating a robust differentiator and an adaptive law. The robust differentiator is used to estimate the velocity and acceleration without requiring the system model. The adaptive law will update the feedback gain online without requiring the disturbance bound. The relatively slow actuator dynamics of the process are accommodated both within the selection of the order of the SMC and within the controller design. In summary, the proposed approach does not require an accurate model or disturbance bound which thus relaxes the design conditions.

Remark 4: In Fig. 1, a robust differentiator [20] is used to estimate the velocity and acceleration online, which does not require the dynamic model and then make the design easier compared with the existing state observers. Its effectiveness will be validated in the case study. 


\section{Robust AdAptive SMC Design}

In this section, an adaptive SMC algorithm will be designed for DP of the SSOP. For (7), the tracking error is defined as

$$
e=\eta-\eta_{d}
$$

where $e \in R^{3}$ is the tracking error vector, $\eta_{d} \in R^{3}$ is the desired position vector.

Assumption 4: $\eta_{d}, \dot{\eta}_{d}$ and $\ddot{\eta}_{d}$ are known and bounded.

The siding mode is defined as

$$
s=\ddot{e}+\Lambda_{1} \dot{e}+\Lambda_{2} e
$$

where $\Lambda_{1}=\operatorname{diag}\left\{\left[\lambda_{1}^{1}, \lambda_{2}^{1}, \lambda_{3}^{1}\right]\right\} \in R^{3}, \Lambda_{2}=$ $\operatorname{diag}\left\{\left[\lambda_{1}^{2}, \lambda_{2}^{2}, \lambda_{3}^{2}\right]\right\} \in R^{3} . \ddot{e}_{i}+\lambda_{i}^{1} \dot{e}_{i}+\lambda_{i}^{2} e_{i}$ should be Hurwitz, $i=1,2,3$.

In the following, a robust SMC will be initially designed for DP of the SSOP, then a corresponding robust adaptive SMC will be developed.

\section{A. Sliding Mode Controller Design}

An SMC can be defined for DP of the SSOP by

$$
\begin{aligned}
u= & u_{e q}+u_{s w} \\
u_{e q}= & \Lambda^{-1}\left[P\left(\dddot{\eta}_{d}-\Lambda_{1} \ddot{e}-\Lambda_{2} \dot{e}\right)+\dot{P} \ddot{\eta}+\right. \\
& \dot{Q} \dot{\eta}+Q \ddot{\eta}+\Lambda P \ddot{\eta}+\Lambda Q \dot{\eta}] \\
u_{s w}= & -\Lambda^{-1} P K \frac{s}{\|s\|}
\end{aligned}
$$

where $K \in R$ and $K>\gamma_{0}$ is a feedback gain, $u_{e q}$ is the equivalent control and $u_{s w}$ is the discontinuous control term, $u$ is the applied control.

Remark 5: In (10), $u_{e q}$ controls the nominal portion of (7), $u_{s w}$ deals with the effects of $d$ and $\dot{d}$ in (7), $K$ is an important control parameter to suppress $d$ and $\dot{d}$.

Theorem 1: For (7), under Assumptions 1-4, the control law (10) will stabilize the tracking error to zero asymptotically if $K>\gamma_{0}$.

Proof:

Select a Lyapunov candidate as

$$
V=\frac{1}{2} s^{T} s
$$

Differentiate (13) with respect to time along (7)

$$
\begin{aligned}
& \dot{V}=s^{T}\left[P^{-1}(\Lambda u-\dot{P} \ddot{\eta}-\dot{Q} \dot{\eta}-Q \ddot{\eta}+\dot{d}\right. \\
& \left.-\Lambda P \ddot{\eta}-\Lambda Q \dot{\eta}+\Lambda d)-\dddot{\eta}_{d}+\Lambda_{1} \ddot{e}+\Lambda_{2} \dot{e}\right]
\end{aligned}
$$

Substitute (10) into (14)

$$
\begin{aligned}
\dot{V} & =-K\|s\|+s^{T} P^{-1} \dot{d}+s^{T} P^{-1} \Lambda d \\
& \leqslant-K\|s\|+\left(\left\|P^{-1}\right\|\|\dot{d}\|+\left\|P^{-1} \Lambda\right\|\|d\|\right) \| s
\end{aligned}
$$

Consider Assumption 3

$$
\dot{V} \leqslant-\left(K-\gamma_{0}\right)\|s\|
$$

If $K$ is appropriately selected and $K-\gamma_{0}>0$ the reaching condition will be satisfied [25]. Then, the tracking error will reach the sliding mode in finite time and converge to 0 asymptotically, that is, $e \rightarrow 0$ as $t \rightarrow \infty$. Q.E.D.

Remark 6: In (12), $K$ is the feedback gain which is also a robust control gain according to (17). One of the control design questions is how to find an appropriate $K$. This requires knowledge of the disturbance bound in advance; alternatively a trial and error method can be employed. In the following subsection a novel adaptive law will be developed to update $K$ online.

\section{B. Robust Adaptive Sliding Mode DP Control}

Assumption 5: Assume there is an appropriate $K>\gamma_{0}$.

To make the algorithm easier to implement, an adaptive law will be designed to estimate the feedback gain. Assume $\hat{K} \in R$ is an estimate of $K$, then the estimation error is defined as

$$
\tilde{K}=K-\hat{K}
$$

where $\tilde{K} \in R$ is the estimation error. Its time differential is given as

$$
\dot{\tilde{K}}=-\dot{\hat{K}}
$$

The following robust adaptive SMC can be designed for DP of the SSOP

$$
\begin{aligned}
u= & u_{e q}+u_{s w} \\
u_{e q}= & \Lambda^{-1}\left[P\left(\dddot{\eta}_{d}-\Lambda_{1} \ddot{e}-\Lambda_{2} \dot{e}\right)+\dot{P} \ddot{\eta}+\right. \\
& \dot{Q} \dot{\eta}+Q \ddot{\eta}+\Lambda P \ddot{\eta}+\Lambda Q \dot{\eta}] \\
u_{s w}= & -\Lambda^{-1} P \hat{K} \frac{s}{\|s\|} \\
\dot{\hat{K}}= & \kappa\|s\|
\end{aligned}
$$

where $\kappa \in R$ and $\kappa>0$ is the update gain.

Theorem 2: For (7), under Assumptions 1-5, the control law (20)-(23) will stabilize the tracking error to zero asymptotically if $\kappa>0$.

Proof:

Select a Lyapunov function candidate as

$$
V=\frac{1}{2} s^{T} s+\frac{1}{2 \kappa} \tilde{K}^{2}
$$

Differentiate (24) with respect to time along (7)

$$
\begin{aligned}
\dot{V}= & s^{T}\left[P^{-1}(\Lambda u-\dot{P} \ddot{\eta}-\dot{Q} \dot{\eta}-Q \ddot{\eta}+\dot{d}-\Lambda P \ddot{\eta}\right. \\
& \left.-\Lambda Q \dot{\eta}+\Lambda d)-\dddot{\eta}_{d}+\Lambda_{1} \ddot{e}+\Lambda_{2} \dot{e}\right]+\frac{1}{\kappa} \tilde{K} \dot{\tilde{K}}(25)
\end{aligned}
$$

Substitute (20) into (25)

$$
\begin{aligned}
\dot{V}= & -\hat{K}\|s\|+s^{T} P^{-1} \dot{d}+s^{T} P^{-1} \Lambda d-\frac{1}{\kappa} \tilde{K} \dot{\hat{K}} \\
\leqslant & -\hat{K}\|s\|+\left(\left\|P^{-1}\right\|\|\dot{d}\|+\right. \\
& \left.\left\|P^{-1} \Lambda\right\|\|d\|\right)\|s\|-\frac{1}{\kappa} \tilde{K} \dot{\dot{K}} K \\
\leqslant & -\hat{K}\|s\|+\gamma_{0}\|s\|-\frac{1}{\kappa} \tilde{K} \dot{\hat{K}}
\end{aligned}
$$

Substitute (23) into (28)

$$
\dot{V} \leqslant-\hat{K}\|s\|+\gamma_{0}\|s\|-\tilde{K}\|s\|
$$


Considering (18) and (19)

$$
\dot{V} \leqslant-\left(K-\gamma_{0}\right)\|s\| \leqslant 0
$$

From Assumption 5, there exists a $K$ such that $K-\gamma_{0}>0$. The reaching condition should be satisfied [25]. Then, motion of $e$ is restricted to $s=0$ and $e \rightarrow 0$ as $t \rightarrow \infty$.

Remark 7: An adaptive law has been developed which can update the feedback gain online. Note that the suggested adaptation law may over estimate the uncertainty bound [23]. This can happen for example in the presence of noisy measurement signals. In order to avoid this situation, the implementation

$$
\dot{\hat{K}}=\left\{\begin{array}{ccc}
\kappa\|s\| & \text { if } & \|s\|>\varepsilon \\
0 & \text { elseif } & \|s\| \leq \varepsilon
\end{array},\right.
$$

where $\varepsilon>0$ is a positive constant, is used. Here, adaption is halted whenever the specified domain of $\|s\|$ of width $\varepsilon$ is reached. Consequently a real sliding motion is established.

\section{CAse Study}

The dynamic parameters of the SSOP are given by

$$
\begin{gathered}
M=\left[\begin{array}{ccc}
5.3122 \times 10^{6} & 0 & 0 \\
0 & 8.2831 \times 10^{6} & 0 \\
0 & 0 & 3.7454 \times 10^{9}
\end{array}\right] \\
D=\left[\begin{array}{ccc}
5.0242 \times 10^{4} & 0 & 0 \\
0 & 2.7229 \times 10^{5} & -4.3933 \times 10^{6} \\
0 & -4.3933 \times 10^{6} & 4.1894 \times 10^{8}
\end{array}\right] \\
d(t)=\left[\begin{array}{c}
7.5 \times 10^{3} \sin (1000 t)+0.9 \times 10^{3} \sin (0.01 t) \\
3.5 \times 10^{3} \sin (1000 t)+1.2 \times 10^{3} \sin (0.01 t) \\
1.5 \times 10^{3} \sin (1000 t)+1.5 \times 10^{3} \sin (0.01 t)
\end{array}\right]
\end{gathered}
$$

where $M$ is the inertia matrix, $D$ is the damping matrix and $d(t)$ is the external disturbance. The desired position, velocity and acceleration are given as $\eta_{d}=[50,50,50]^{T}$, $\dot{\eta}_{d}=[0,0,0]^{T}, \ddot{\eta}_{d}=[0,0,0]^{T}$, the initial value of the SSOP is given as $\eta(0)=[0,0,0]^{T}$ and $\dot{\eta}(0)=[0,0,0]^{T}$. The actuator characteristics are defined by: $\Lambda=\operatorname{diag}([25,25,25])$, $\Lambda_{1}=\operatorname{diag}([5,5,5]), \Lambda_{2}=\operatorname{diag}([5,5,5])$

The controller parameters are selected as follows, (1) Robust SMC for DP of SSOP $K=10$; (2) Robust Adaptive SMC for DP of SSOP: $\kappa=0.0008, \hat{K}(0)=0, \varepsilon=10$.

Remark 8: A novel robust differentiator was used to estimate the velocity and acceleration measurements which are required for the proposed SMC design. Full details are available at [20].

\section{Case 1: Robust SMC for DP of SSOP}

The performance of the robust SMC was demonstrated in the presence of external disturbances. Fig. 2-3 show the position tracking accuracy and the torque inputs applied to the platform. In Fig. 2, the SSOP is seen to track the desired position well despite the presence of the external disturbance. The torque signals applied to the SSOP are sufficiently smooth enough and bounded ( Fig. 3). The robust SMC has achieved good performance for DP of SSOP. However the feedback gain $K$ was chosen carefully because it plays an important role on the system stability and robustness. The designer needs to know the external disturbance bound for the controller design, which is not straightforward in practice. A trial and error method is often used to find an appropriate $K$. On site, this requires that the operator has a wealth of experience and can be time-consuming.
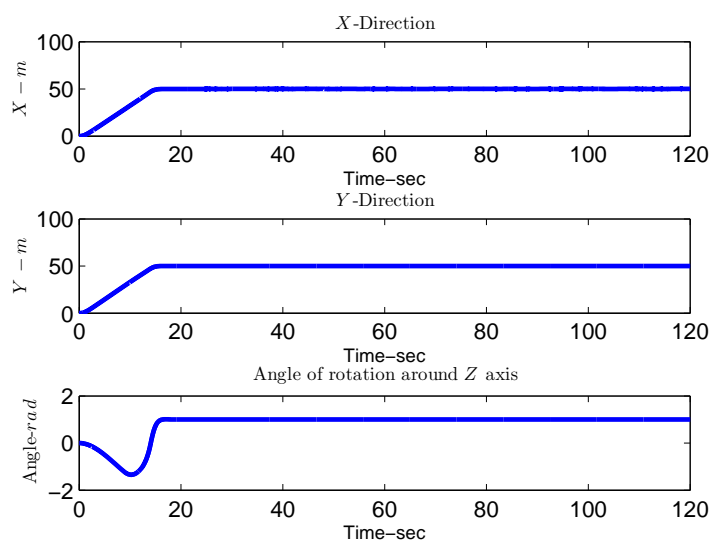

Fig. 2. The position tracking performance of the robust SMC strategy.
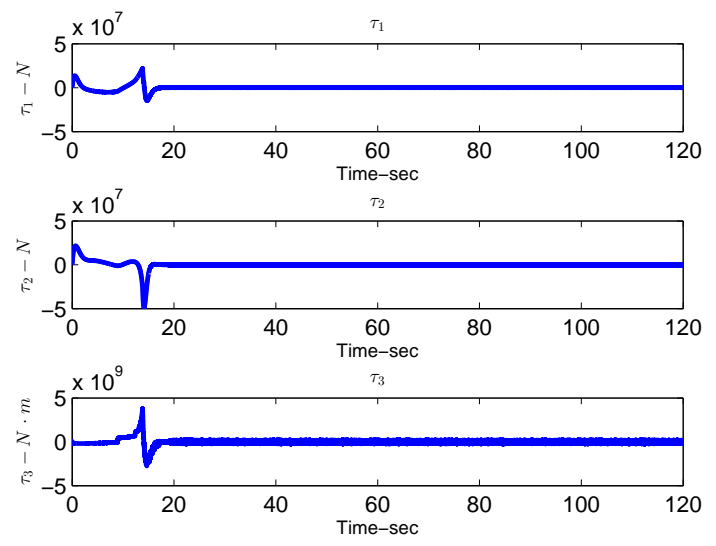

Fig. 3. The torque inputs from the robust SMC strategy.

\section{Case 2: Robust Adaptive SMC for DP of SSOP}

The performance of the robust adaptive SMC is demonstrated in the presence of an external disturbance. Fig. 4-6 show the position tracking, the torque inputs and the adaptive law, respectively. In Fig. 4, the SSOP can track the desired position despite the external disturbance. The torque inputs applied to the SSOP are smooth enough and bounded in Fig. 5. The adaptive law is shown in Fig. 6; this updates the feedback control gain $K$ online. Because (31) is used in the implementation, the adaptive law does not exhibit over estimation. Here, the bound on the disturbance is not known a priori. Comparing Cases 1 and 2, the control performance of the robust adaptive SMC is as good as the robust SMC. These 
results further validate the effectiveness of the proposed robust adaptive SMC.
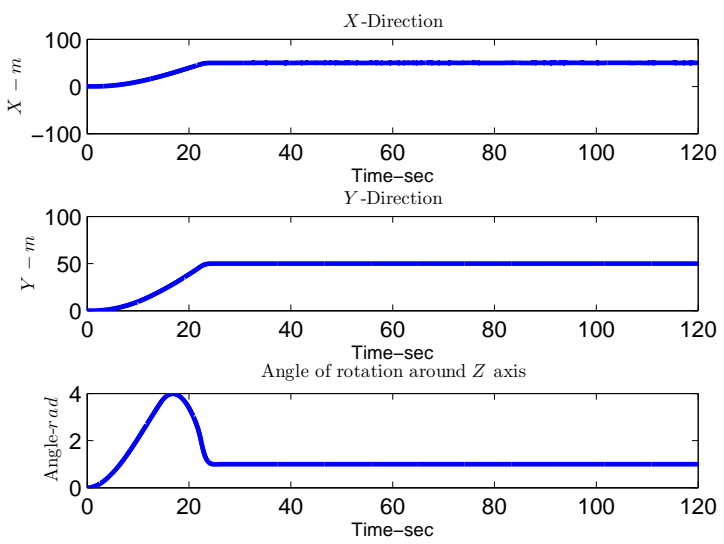

Fig. 4. The position tracking performance of the robust adaptive SMC for DP of SSOP.
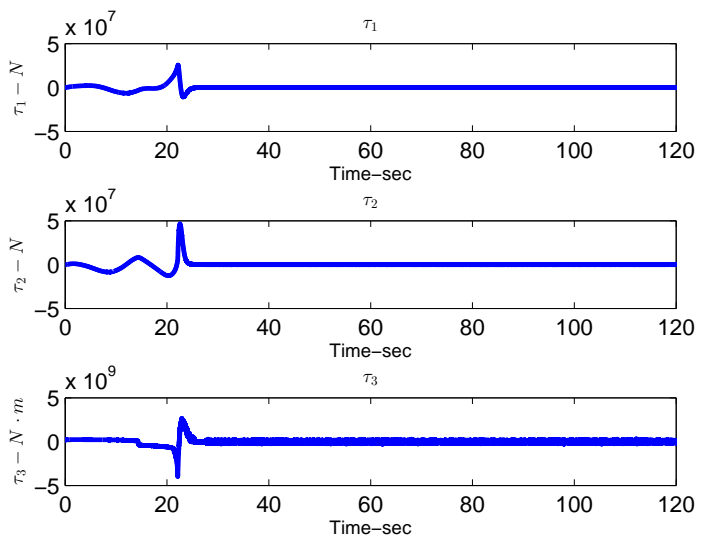

Fig. 5. The torque inputs from the robust adaptive SMC.

Case 3: Robust Adaptive SMC for DP of SSOP in the Presence of an Impulsive External Disturbance

To further validate the approach, an impulsive external disturbance $\left[7 \times 10^{6} \mathrm{~N}, 7 \times 10^{6} \mathrm{~N}, 7 \times 10^{6} \mathrm{~N} \cdot \mathrm{m}\right]^{T}$ is added to the simulation during the period $70 \mathrm{sec} \leq t \leq 75 \mathrm{sec}$. Fig. 7-9 show the position tracking performance, the applied torque input and the adaptive law, respectively. From Fig. 7 , the impulsive disturbance affects the position tracking but the proposed approach can attenuate the impact of the disturbance. From Fig. 8 and 9, the applied torque input and adaptive law are smooth and bounded. The performance is verified in the presence of a strong impulsive disturbance.

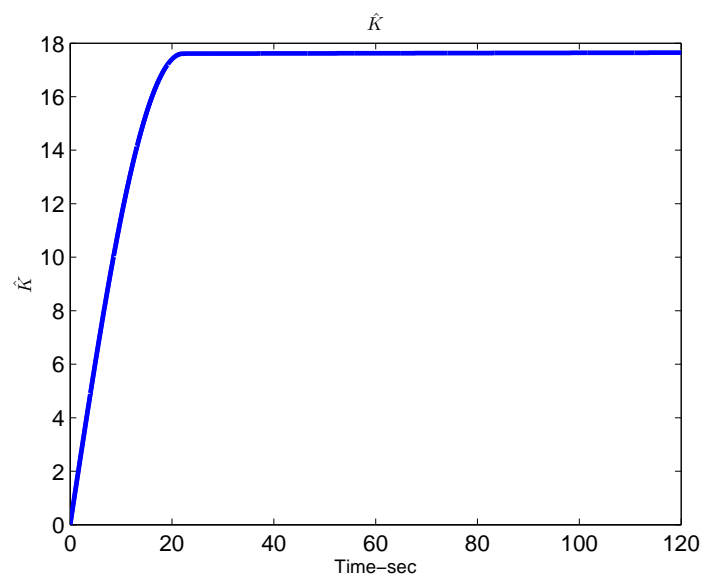

Fig. 6. The adaptive law of the robust adaptive SMC.
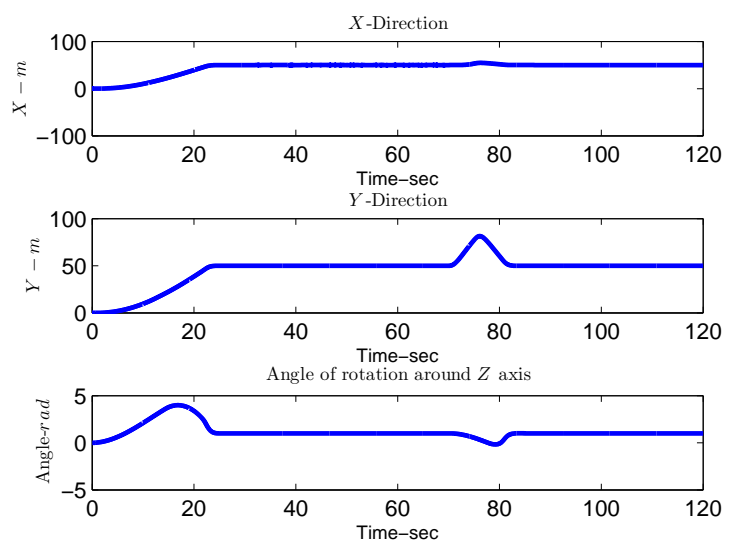

Fig. 7. The position tracking performance of the robust adaptive SMC.

\section{Conclusions}

Using adaptation coupled with a robust differentiator, a novel robust adaptive SMC has been developed to achieve dynamic positioning for a semi-submersible offshore platform. The proposed approach achieves asymptotic stability and is seen to provide an effective dynamic positioning solution which exhibits good robustness. Due to the use of the differentiator, velocity and acceleration can be obtained online without requiring the system model. The adaptation which updates feedback control gain is an implementable solution. The methodology accommodates the actuator dynamics, which in this application domain are relatively slow. Stability analysis and numerical simulation results have been presented to support the arguments presented in the article. Future work will focus on the implementation in a practical system.

\section{REFERENCES}

[1] Hu X, Du J, Sun Y. Robust Adaptive Control for Dynamic Positioning of Ships. IEEE Journal of Oceanic Engineering, 2017, (99):1-10. 

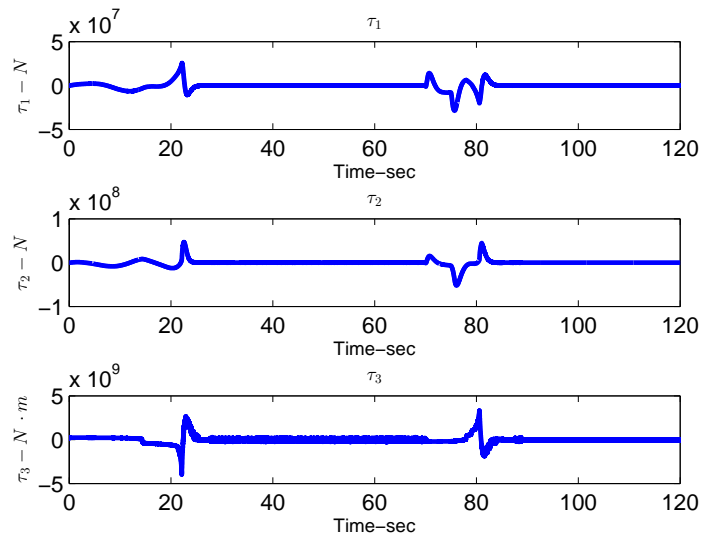

Fig. 8. The applied torque from the robust adaptive SMC.

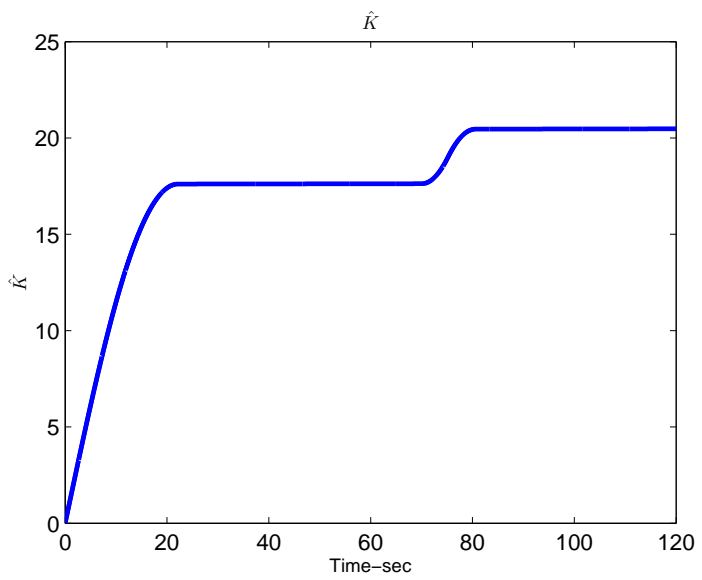

Fig. 9. The adaptive law for the robust adaptive SMC.

[2] Xu D, Xia S, Zhang H, Wu Y. Adaptive optimal control of multimodular floating platforms in random seas. Nonlinear Dynamics, 2017, 91(2): 863-876.

[3] S $\phi$ rensen A J. A survey of dynamic positioning control systems. Annual Reviews in Control, 2011, 35(1): 123-136.

[4] Yamamoto M, Morooka C K. Dynamic positioning system of semisubmersible platform using fuzzy control. Journal of the Brazilian Society of Mechanical Sciences \& Engineering, 2005, 27(4): 449-455.

[5] S $\phi$ rensen A J, Sagatun S I, Fossen T I, Design of a dynamic positioning system using model-based control. Control Engineering Practice, 1996, 4(3): 359-368.
[6] Chen H, Wan L, Wang F, Zhang G. Model predictive controller design for the dynamic positioning system of a semi-submersible platform. Journal of Marine Science and Application, 2012, 11(3): 361-367.

[7] Che M, Ge S S, How B V E, Choo Y S. Robust adaptive position mooring control for marine vessels. IEEE Trans. on Control Systems Technology, 2013, 21(2): 395-409.

[8] Muhammad S, Dòria-Cerezo A. Passivity-based control applied to the dynamic positioning of ships. IET control theory \& applications, 2012, 6(5): 680-688.

[9] Godhavn J-M, Fossen T I, Berge S P. Non-linear and adaptive backstepping designs for tracking control of ships. International Journal of Adaptive Control and Signal Processing, 1998, 12(8): 649-670.

[10] Tannuri E A, and Agostinho A C, Morishita H M, Moratelli J L. Dynamic positioning systems: An experimental analysis of sliding mode control. Control Engineering Practice, 2010, 18(10): 1121-1132.

[11] Tannuri E A, Donha D C, Pesce C P. Dynamic positioning of a turret moored fpso using sliding mode control. International Journal of Robust \& Nonlinear Control, 2001, 11(13): 1239-1256.

[12] Ashrafiuon H, Muske K R, Mcninch L C, Soltan R A. Slidingmode tracking control of surface vessels. IEEE Trans. on Industrial Electronics, 2008, 55(11): 4004-4012.

[13] Valenciaga F. A second order sliding mode path following control for autonomous surface vessels. Asian Journal of Control, 2014, 16(5): $1515-1521$

[14] Levant A., Fridman, L.M., Accuracy of Homogeneous Sliding Modes in the Presence of Fast Actuators, IEEE Trans. on Automatic Control, 2010, 55(3): 810-814.

[15] Utkin V., Discussion Aspects of High-Order Sliding Mode Control, IEEE Trans. on Automatic Control, 2016, 61(3): 829-833.

[16] Fridman, L., Is it reasonable to discontinuous SMC by continuous HOSMC, 2017, Proceedings of the Styrian Workshop on Automatic Control (eds. Steinberger M, Horn M), ISBN: 978-3-901439-12-4

[17] Du J, Hu X, Liu H, Chen C L. Adaptive robust output feedback control for a marine dynamic positioning system based on a highgain observer. IEEE Trans. Neural Networks and Learning Systems, 2015, 26(11): 2775-2786.

[18] Fossen T I, Strand J P. Passive nonlinear observer design for ships using Lyapunov methods: full-scale experiments with a supply vessel. Automatica, 1999, 35(1): 3-16.

[19] Xu D, Du J, Hu X, Li H. Sliding mode observer design for ship dynamic positioning system. Fifth International Conference on Intelligent Control and Information Processing, Dalian, China, 2014, 151-154.

[20] Reichhartinger M, Spurgeon S K. A robust exact differentiator block for matlab/simulink. ResearchGate Technical Report, 2016, DOI: 10.13140/RG.2.1.3243.4803, pp. 1C10.

[21] Reichhartinger M, Spurgeon S K. An arbitrary-order differentiator design paradigm with adaptive gains, International Journal of Control, 2018, https://doi.org/10.1080/00207179.2018.1429671

[22] Bandyopadhyay B, Janardhanan S, Spurgeon S. Advances in sliding mode control: concept, theory and implementation, Springer Berlin, 2013.

[23] Plestan F, Shtessel Y, Bregeault V, Poznyak A. New methodologies for adaptive sliding mode control. International Journal of Control, 2010, 83(9): 1907-1919.

[24] Fossen T I. Handbook of Marine Craft Hydrodynamics and Motion Control, John Wiley, 2011.

[25] Slotine J-J E, Li W. Applied nonlinear control, Prentice Hall, 1991. 